\title{
Severe Aplastic Anemia and PNH
}

\author{
Régis Peffault de Latour, Antonio Risitano, \\ and Carlo Dufour
}

\subsection{Definition and Epidemiology}

Severe aplastic anemia (SAA) is an autoimmune disorder (AID) due to the attack of autoreactive cytotoxic $\mathrm{T}$ lymphocytes to the hematopoietic component of the bone marrow. The triggering antigen is so far unknown. The incidence of SAA is about 2.34/million in Europe and the United States and threefold higher in East Asia, with two age peaks of incidence (in young adults and in the elderly) (Young and Kaufman 2008).

Paroxysmal nocturnal hemoglobinuria (PNH) is another bone marrow failure syndrome (BMFS) which is often embedded with SAA. PNH is a more heterogeneous disease since its clinical presentation includes hemolytic anemia and thrombophilia in addition to bone marrow failure.

R. P. de Latour

French Reference Center for Aplastic Anemia and Paroxysmal Nocturnal Hemoglobinuria, Saint-Louis Hospital, Paris, France

Assistance Publique-Hôpitaux de Paris, Saint-Louis Hospital, Paris, France

University Paris VII, Denis Diderot, Paris, France

A. Risitano

Department of Clinical Medicine and Surgery, Federico II University of Naples, Naples, Italy

C. Dufour $(\bowtie)$

Hematology Unit, G. Gaslini Research Children

Hospital, Genoa, Italy

e-mail: carlodufour@gaslini.org

\subsection{Diagnosis and Indication for Treatment for SAA}

SAA is usually diagnosed in the setting of pancytopenia and a hypocellular BM. Diseases such as myelodysplasia, myelofibrosis, hypocellular acute leukemia, inherited BMF such as Fanconi's anemia (FA), or telomeropathies need to be excluded. Cytogenetic abnormalities can be found in up to $10 \%$ of true SAA (Rovo et al. 2016; Barone et al. 2015).

There is a close relationship between PNH and acquired SAA with a concomitant diagnosis in $40 \%$ of cases. SAA is diagnosed when marrow hematopoietic cellularity is $<30 \%$, and two of three of the following criteria are met: absolute neutrophil count $<0.5 \times 10^{9} / \mathrm{L}$, absolute reticulocyte count $<60 \times 10^{9} / \mathrm{L}$, and platelet count $<20 \times 10^{9} / \mathrm{L}$ (Camitta et al. 1976).

Treatment requires careful planning and may be prolonged. A watch and wait strategy is often used initially if there is milder pancytopenia. Conversely, in case of transfusion requirement or if the criteria for SAA are met, treatment should begin with no delay. Prior to treatment the patient should be stable clinically with control of bleeding and infections. Once the diagnosis is confirmed, and the disease severity is assessed, family HLA-typing and matched unrelated donor search should be done in the work-up phase. In the absence of sign of intravascular hemolysis, patient's treatment algorithm is similar with or without PNH. 


\subsection{Treatment of SAA}

\subsubsection{First Line Treatment for SAA}

The choice of first-line treatment depends on the age of the patient and the availability of an HLA MSD (Fig. 77.1). The standard first-line treatments for a newly diagnosed patient with SAA are HSCT from a HLA-identical sibling donor or IS therapy (IST) using a combination of horse ATG and CSA (ATG + CSA). Early bone marrow HSCT after a conditioning regimen with CY, ATG, and GVHD prophylaxis combining CSA and MTX promotes excellent engraftment (95\%) and OS (90\% at 2 years) (Bacigalupo et al. 2012; Peffault de Latour 2016). This approach enabled also a very good long-term outcome with a rather limited number of late effects consisting in avascular necrosis, endocrine dysfunctions, and very rare secondary malignancy (Konopacki et al. 2012). However, toxicity related to transplantation as well as increased risk of GvHD is still a problem for patients older than 40 years of age and for those with high comorbidity index (Marsh et al. 2011).

For these categories first-line IS with horse ATG + CSA is recommended. This combination showed both in prospective controlled studies (Scheinberg et al. 2011; Marsh et al. 2012) and in real-life surveys (Peffault de Latour et al. 2018) a response rate of about $60 \%$. Frontline IST provided similar findings in children and adolescents (Dufour et al. 2014; Dufour et al. 2015a). After IST, responders might experience relapse (20$30 \%$ ), CSA dependence (20-30\%), or long-term clonal evolution (PNH, MDS, or AML) (Scheinberg and Young 2012), justifying regular follow-up and bone marrow evaluation every 12-18 months.

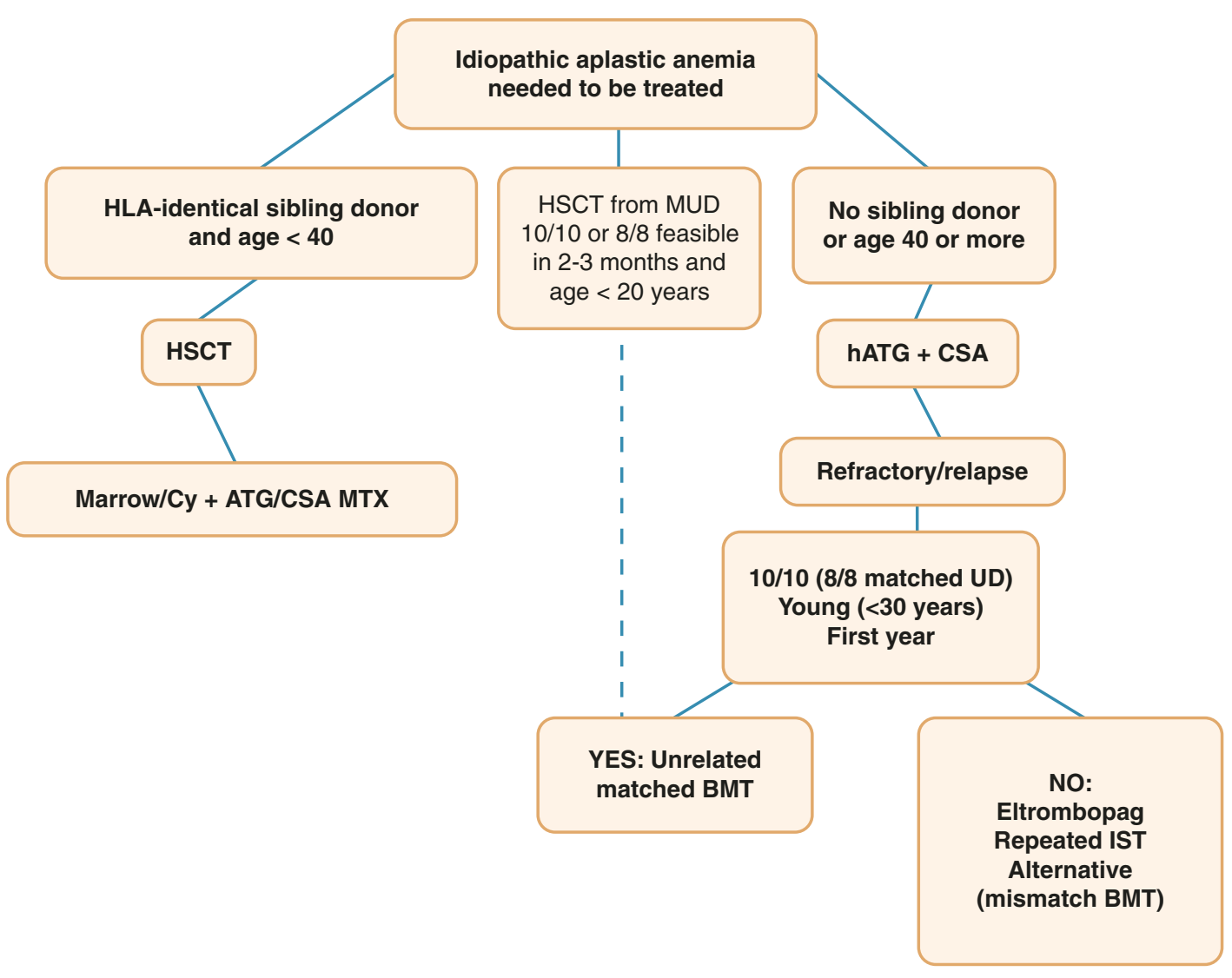

Fig. 77.1 Treatment algorithm of SAA in 2019 


\subsubsection{Second-Line Treatment for SAA}

The choice of second-line treatment is also driven by age, by comorbidities, and by the presence of a matched related (MRD) or unrelated donor (MUD):

- In older patients with a MRD and confirmed refractory SAA, HSCT should be considered in the absence of significant comorbidities.

- In younger patients with a MUD and refractory or relapsed SAA, HSCT is recommended. Results of MUD HSCT have improved to such an extent that OS of idiopathic SAA are not statistically inferior to MRD transplants (Bacigalupo et al. 2015). This improvement has been largely attributed to better donor selection through allele matching, progress in supportive care, prophylaxis of GVHD, incorporation of FLU in conditioning regimens, and the addition of lowdose TBI. Recently some factors were found to positively affect OS after MUD HSCT including age $\leq 30$ years, transplant within the first year after diagnosis (Devillier et al. 2016), use of BM vs PB, and CMV status (Bacigalupo et al. 2015).

- For patients older than 30 years, monotherapy with eltrombopag, an oral thrombopoietinreceptor agonist, produced in prospective studies in refractory patients an overall response of $40 \%$ with trilineage responses in some cases (Olnes et al. 2012; Desmond et al. 2014). A retrospective French study found similar results on patients with relapsed/ refractory SAA. The overall rates of red blood cell and platelet transfusion independence were $7 \%, 33 \%, 46 \%$, and 46 at 1, 3, and 6, months and last follow-up, respectively. No clonal evolution has been documented so far (Lengline et al. 2018). Other second-line options for patients not eligible to HSCT and who relapse or do not respond to frontline IST are a second course of ATG (rabbit) + CSA and alemtuzumab offering a response rate of $65 \%$ (Scheinberg et al. 2006) and 37\% (Scheinberg and Young 2012), respectively.

\subsubsection{Emerging Strategies for SAA}

\subsubsection{Eltrombopag Added to the Standard Horse ATG + CSA First Line Treatment}

Many efforts to improve results of the standard treatment with horse ATG and CSA have failed since 40 years (Scheinberg 2012). Excellent results obtained with eltrombopag in monotherapy in refractory patients prompted American colleagues from the NIH to test if the addition of eltrombopag to standard IST as the first treatment for SAA would have increased the rate of CR and improved the long-term outcome. In the best cohort (eltrombopag associated to ATG and CSA from day 1), complete and overall response rates at 6 months were $58 \%$ and $94 \%$, respectively. After a median follow-up of 2 years, survival rate is $97 \%$ (Townsley et al. 2017). Rates of relapse and clonal evolution were similar to historical experience. Whether eltrombopag might substantially improve horse ATG + CSA platform is at the moment under investigation through a large, randomized, controlled, prospective European trial on behalf of the SAA working party of the EBMT (RACE trial; ClinicalTrials.gov number, NCT02099747).

\subsubsection{Up-Front Matched Unrelated Donor Transplantation}

Although pediatric patients respond better to IST, the long-term risks of relapse, CSA dependence, and clonal evolution are high (Dufour et al. 2014). UK investigators reported an excellent estimated 5-year FFS of $95 \%$ in 44 consecutive children who received a 10-antigen (HLA-A, HLA-B, HLA-C, HLA-DRB1, HLA-DQB1) MUD HSCT; 40 of these children had previously failed IST. HSCT conditioning was with FLU, CY, and campath (FCC) (Samarasinghe and Webb 2012). Because of those excellent results, up-front MUD HSCT became an attractive firstline option in children. Between 2005 and 2014, a UK cohort of 29 consecutive children with idiopathic SAA received UD HSCTs (including five patients with $1 \mathrm{Ag}$ mismatched transplants) as 
first-line therapy after conditioning with FCC. Results were excellent, with OS and EFS of $96 \%$ and $92 \%$, respectively, low GVHD rates, and only one death (from idiopathic pneumonia) (Dufour et al. 2015b). This cohort was then compared with historical matched controls who had received (1) first-line MRD HSCT, (2) first-line IST with horse ATG + CSA, and (3) MUD HSCT post-IST failure as second-line therapy. Outcomes for the up-front unrelated cohort were similar to MRD HSCT and superior to IST and UD HSCT post-IST failure. Similar results were observed in another pediatric study (Choi et al. 2017).

Currently a North American study aims to compare outcomes of children with SAA treated de novo with IST vs MUD HSCT (ClinicalTrials. gov number NCT02845596). While waiting the results of this trial, if a 10/10 MUD is available and the transplant appears feasible within 2-3 months since diagnosis, this type of HSCT has become a reasonable frontline option for young patients in many centers. Another option is to perform MUD HSCT early after failure of frontline IST within 4-6 months since diagnosis. This is why MUD donor search should be started at diagnosis in young patients who lack a MRD.

\subsubsection{Alternative Donor Transplantation in SAA}

Alternative HSCTs (MMURD, CB, and haplofamily donors) are possible for individuals with no suitable MUD. Alternative HSCTs may be curative, but the risks of graft rejection, infectious complications, and GVHD are higher than those for MRD or MUD HSCT. Patient age, comorbidities, and alternative HSCT specificities are thus important issues in the decision-making process. Age and comorbidities are the first barriers to this type of procedure. Most numerous cohorts ( $>50$ patients) tend to mainly include pediatric patients. In older studies long-term OS of about 60\% (Yagasaki et al. 2011; Horan et al. 2012; Peffault de Latour et al. 2011)) compared to 5-year OS seen in refractory patients receiving only supportive care (Valdez et al. 2011). More recent studies with shorter follow-up showed OS and EFS $>80 \%$ in unmanipulated haplo-HSCT with a high rate of cGVHD greater than $30 \%$ (Xu et al. 2016, 2017).
Based on this, alternative HSCT can be considered a salvage option that needs to be carefully balanced with best supportive care. The latter might be preferable for patients with comorbidities or advanced age ( $>40$ years or older) because of lower risks.

\subsection{Treatment of PNH}

Clinical presentation of PNH is extremely heterogeneous, including a variable combination of bone marrow failure, hemolytic anemia, and thromboembolism (Peffault de Latour et al. 2008). These clinical manifestations may change during the disease course of each individual patient so that the treatment of $\mathrm{PNH}$ should target the specific clinical presentation (Risitano 2017).

The treatment of marrow failure in PNH parallels that of SAA, and it has been described above; indeed, the presence of a PNH clone does not change the treatment algorithm of SAA.

In contrast, the treatment of complementmediated hemolytic anemia and of thromboembolic PNH is based on complement inhibition through the anti-C5 MoAb eculizumab. Eculizumab has proven to be effective in inhibiting intravascular hemolysis of $\mathrm{PNH}$, leading to hemoglobin stabilization and transfusion independency in about half of patients (Hillmen et al. 2006; Brodsky et al. 2008). This dramatic effect on intravascular hemolysis, eventually resulting in improved quality of life, is also associated with a significant reduction of the risk of thromboembolic complications (Hillmen et al. 2007). Notably, eculizumab treatment leads to a significant improvement of overall survival of $\mathrm{PNH}$ patients, as documented by two independent long-term retrospective studies showing 5-year survival rates $>90 \%$ (Kelly et al. 2011; Loschi et al. 2016). Based on this, eculizumab is currently the standard of care for all PNH patients presenting with symptomatic hemolytic and/or thromboembolic disease; occasionally, when this occurs concomitantly with a BMF, the anticomplement treatment may be considered also in combination with IST (i.e., sequential or concomitant treatment) (Pagliuca et al. 2018). 


\subsubsection{Emerging Strategies for PNH}

Even if currently available anticomplement treatment addresses most clinical needs of patients with hemolytic (and thrombotic) $\mathrm{PNH}$, a number of novel strategies of complement modulation are in their preclinical or clinical development (Risitano and Marotta 2016). These strategies may target specific unmet clinical needs pertaining PNH patients. Novel anti-C5 agents (either MoAb, small molecules or small interfering RNA) may represent an improvement of current eculizumab, mostly in terms of patient comfort due to long-lasting activity (with longer dosing interval) and/or self-administration (usually SC). In addition, a novel class of compounds targeting early steps of the complement cascade at the level of $\mathrm{C} 3$ or even upstream (inhibitors of complement factor B and factor B) may anticipate a better efficacy in terms of hematological response, due to possible effect on C3-mediated extravascular hemolysis. Ongoing clinical trials will reveal whether any of this strategy may lead to change in the standard of care of anticomplement treatment of hemolysis (and thrombophilia) of PNH patients (Risitano and Marotta 2018).

\section{Key Points}

- SAA is usually diagnosed in the setting of pancytopenia and a hypocellular BM when other diseases, especially inherited BMF such as Fanconi's anemia or telomeropathy, have been excluded.

- The preferred treatment of SAA is HSCT from HLA-identical sibling donor. Transplantation from a MUD may be considered for patients without a sibling donor after failure of IS therapy or up front in younger $\leq 20$ years if feasible in $2-3$ months since diagnosis.

- Eltrombopag might substantially change in the coming years the standard horse ATG + CSA platform.

- The role of alternative donor HSCT needs further validation to enter the current clinical practice.

\section{References}

Bacigalupo A, Boyd A, Slipper J, et al. Foscarnet in the management of cytomegalovirus infections in hematopoietic stem cell transplant patients. Expert Rev AntiInfect Ther. 2012;10:1249-64.

Bacigalupo A, Socié G, Hamladji RM, et al. Current outcome of HLA identical sibling versus unrelated donor transplants in severe aplastic anemia: an EBMT analysis. Haematologica. 2015;100:696-702.

Barone A, Lucarelli A, Onofrillo D, et al. Diagnosis and management of acquired aplastic anemia in childhood. Guidelines from the Marrow Failure Study Group of the Pediatric Haemato-Oncology Italian Association (AIEOP). Blood Cells Mol Dis. 2015;55:40-7.

Brodsky RA, Young NS, Antonioli E, et al. Multicenter phase III study of the complement inhibitor eculizumab for the treatment of patients with paroxysmal nocturnal hemoglobinuria. Blood. 2008;114:1840-7.

Camitta BM, Thomas ED, Nathan DG, et al. Severe aplastic anemia: a prospective study of the effect of early marrow transplantation on acute mortality. Blood. 1976;48:63-70.

Choi YB, Yi ES, Lee JW, et al. Immunosuppressive therapy versus alternative donor hematopoietic stem cell transplantation for children with severe aplastic anemia who lack an HLA-matched familial donor. Bone Marrow Transplant. 2017;52:47-52.

Desmond R, Townsley DM, Dumitriu B, et al. Eltrombopag restores trilineage hematopoiesis in refractory severe aplastic anemia that can be sustained on discontinuation of drug. Blood. 2014;123:1818-25.

Devillier R, Dalle JH, Kulasekararaj A, et al. Unrelated alternative donor transplantation for severe acquired aplastic anemia: a study from the French Society of Bone Marrow Transplantation and Cell Therapies and the EBMT Severe Aplastic Anemia Working Party. Haematologica. 2016;101:884-90.

Dufour C, Pillon M, Passweg J, et al. Outcome of aplastic anemia in adolescence: a survey of the Severe Aplastic Anemia Working Party ofthe European Group for Blood and Marrow Transplantation. Haematologica. 2014;99:1574-81.

Dufour C, Pillon M, Sociè G, et al. Outcome of aplastic anaemia in children. A study by the severe aplastic anaemia and paediatric disease working parties of the European group blood and bone marrow transplant. $\mathrm{Br}$ J Haematol. 2015a;169:565-73.

Dufour C, Veys P, Carraro E, et al. Similar outcome of upfront-unrelated and matched sibling stem cell transplantation in idiopathic paediatric aplastic anemia. A study on behalf of the UK BMT WP, Paediatric Diseases and Severe Aplastic Anemia Working Party of EBMT. Br J Haematol. 2015b;171:585-94.

Hillmen P, Young NS, Schubert J, et al. The complement inhibitor eculizumab in paroxysmal nocturnal hemoglobinuria. N Engl J Med. 2006;355:1233-43.

Hillmen P, Muus P, Duhrsen U, et al. Effect of the complement inhibitor eculizumab on thromboembolism in 
patients with paroxysmal nocturnal hemoglobinuria. Blood. 2007;110:4123-8.

Horan J, Wang T, Haagenson M, et al. Evaluation of HLA matching in unrelated hematopoietic stem cell transplantation for nonmalignant disorders. Blood. 2012;120:2918-24.

Kelly RJ, Hill A, Arnold LM, et al. Long-term treatment with eculizumab in paroxysmal nocturnal hemoglobinuria: sustained efficacy and improved survival. Blood. 2011;117:6786-92.

Konopacki J, Porcher R, Robin M, et al. Long-term follow up after allogeneic stem cell transplantation in patients with severe aplastic anemia after cyclophosphamide plus antithymocyte globulin conditioning. Haematologica. 2012;97:710-6.

Lengline E, Drenou B, Peterlin P, et al. Nationwide survey on the use of eltrombopag in patients with severe aplastic anemia: a report on behalf of the French Reference Center for Aplastic Anemia. Haematologica. 2018;103:212-20.

Loschi M, Porcher R. Barraco et al. Impact of eculizumab treatment on paroxysmal nocturnal hemoglobinuria: A treatment versus no-treatment study. Am J Hematol. 2016;91:366-70.

Marsh JC, Gupta V, Lim Z, et al. Alemtuzumab with fludarabine and cyclophosphamide reduces chronic graft-versus-host disease after allogeneic stem cell transplantation for acquired aplastic anemia. Blood. 2011;118:2351-7.

Marsh JC, Bacigalupo A, Schrezenmeier H, et al. Prospective study of rabbit antithymocyte globulin and cyclosporine for aplastic anemia from the EBMT Severe Aplastic Anaemia Working Party. Blood. 2012;119:5391-6.

Olnes MJ, Scheinberg P, Calvo KR, et al. Eltrombopag and improved hematopoiesis in refractory aplastic anemia. N Engl J Med. 2012;367:11-9.

Pagliuca S, Risitano AM, De Fontbrune FS, et al. Combined intensive immunosuppression and eculizumab for aplastic anemia in the context of hemolytic paroxysmal nocturnal hemoglobinuria: a retrospective analysis. Bone Marrow Transplant. 2018;53:105-7.

Peffault de Latour R, Mary JY, Salanoubat C, et al. Paroxysmal nocturnal hemoglobinuria: natural history of diseases ubcategories. Blood. 2008;112:3099-106.

Peffault de Latour R, Purtill D, Ruggeri A, et al. Influence of nucleated cell dose on overall survival of unrelated cordbloodtransplantation for patients with severe acquired aplastic anemia: a study by eurocordand the aplastic anemia working party of the European group for blood and marrow transplantation. Biol Blood Marrow Transplant. 2011;17:78-85.

Peffault de Latour R. Transplantation for bone marrow failure: current issues. Hematology Am Soc Hematol Educ Program. 2016;2016(1):90-8.
Peffault de Latour R, Tabrizi R, Marcais A, et al. Nationwide survey on the use of horse antithymocyte globulins (ATGAM) in patients with acquired aplastic anemia: A report on behalf of the French Reference Center for Aplastic Anemia. Am J Hematol. 2018;93:635-42.

Risitano AM, Marotta S. Therapeutic complement inhibition in complement-mediated hemolytic anemias: Past, present and future. Semin Immunol. 2016;28:223-40.

Risitano AM. In: Aljurf M, Gluckman E, Dufour C, editors. Congenital and acquired bone marrow failure. New York: Elsevier Inc; 2017. p. 153-64.

Risitano AM, Marotta S. Toward complement inhibition 2.0: Next generation anticomplement agents for paroxysmal nocturnal hemoglobinuria. Am J Hematol. 2018;93:564-77.

Rovo A, et al. Current approach for the diagnosis of Aplastic Anemia. Curr Drug Targets. 2016;17:1.

Samarasinghe S, Webb DK. How I manage aplastic anaemia in children. Br J Haematol. 2012;157:26-40.

Scheinberg P, Nunez O, Young NS. Retreatment with rabbit anti-thymocyte globulin and cyclosporin for patients with relapsed or refractory severea plastic anaemia. Br J Haematol. 2006;133:622-7.

Scheinberg P, Nunez O, Weinstein B, et al. Horse versus rabbit antithymocyte globulin in acquired aplastic anemia. N Engl J Med. 2011;365:430-8.

Scheinberg P, Young NS. How I treat acquired aplastic anemia. Blood. 2012;120:1185-96.

Scheinberg P. Aplastic anemia: therapeutic updates in immunosuppression and transplantation. Hematology Am Soc Hematol Educ Program. 2012;2012:292-300.

Townsley DM, Scheinberg P, Winkler T, et al. Eltrombopag added to standard immunosuppression for aplastic anemia. N Engl J Med. 2017;376:1540-50.

Valdez JM, Scheinberg P, Nunez O, et al. Decreased infection-related mortality and improved survival in severe aplastic anemia in the past two decades. Clin Infect Dis. 2011;52:726-35.

Xu LP, Wang SQ, Wu DP, et al. Haplo-identical transplantation for acquired severe aplastic anaemia in a multicentre prospective study. $\mathrm{Br} \mathrm{J}$ Haematol. 2016;175:265-74.

Xu LP, Zhang XH, Wang FR, et al. Haploidentical transplantation for pediatric patients with acquired severe aplastic anemia. Bone Marrow Transplant. 2017;52:381-7.

Yagasaki H, Kojima S, Yabe H, et al. Acceptable HLAmismatching in unrelated donor bone marrow transplantation for patients with acquired severe aplastic anemia. Blood. 2011;118:3186-90.

Young NS, Kaufman DW. The epidemiology of acquired aplastic anemia. Haematologica. 2008;93:489-92. 
Open Access This chapter is licensed under the terms of the Creative Commons Attribution 4.0 International License (http://creativecommons.org/licenses/by/4.0/), which permits use, sharing, adaptation, distribution and reproduction in any medium or format, as long as you give appropriate credit to the original author(s) and the source, provide a link to the Creative Commons license and indicate if changes were made.

The images or other third party material in this chapter are included in the chapter's Creative Commons license, unless indicated otherwise in a credit line to the material. If material is not included in the chapter's Creative Commons license and your intended use is not permitted by statutory regulation or exceeds the permitted use, you will need to obtain permission directly from the copyright holder.

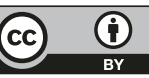

